\title{
Possibilities of Planned Small Families in Communities with Lack of Awareness and Scarce Resources
}

\section{Abstract}

Each day, more than 800 women die due to pregnancy, birth related causes. Most deaths are preventable through birth control, prevention of unwanted pregnancies and increased interval between pregnancies.

Objectives: To get information related to possibilities of small families in communities with extreme poverty with mission of future research and advocacy for small families.

Methodology: Information was collected by simple review of relevant literature, studies, reviews, commentaries. Personal observations were added.

Evidence: It is not very well known whether people with extreme poverty really want to have large families while living with resource crunch, which makes their survival difficult. Rich-poor gap in contraceptive use is large, because of gap in use of modern spacing methods which is much higher in rich compared to poor. Women from poor families tend to have more children, as they give birth at young age, continue childbearing beyond 40 and are less likely to use contraceptive with more unmet need. High fertility has significant implications on welfare of family. Burden of large family perpetuates vicious cycle of poverty, less contraception, more children and more poverty. Reasons given by respondents for non-use of contraception suggest that accessibility of family planning services is not always a barrier even among poorest and there are other factors which result in relatively low use. Women who receive comprehensive factual family planning education are more likely to use contraceptives. Contraceptives with greater side effects or negative cultural perceptions are less likely to be used. Unmet need of contraception due to lack of resolve to practice contraception is not known.

Possibilities: It is essential to know whether couples/women with extreme poverty ever think about planned small family. If no, why, if yes, why action is not taken. Providing culturally sensitive, family centered, accessible sustainable system is essential. There is need of women centered environment that respects their beliefs and helps them have small, planned families.

Keywords: Women; Birth control; Pregnancy; Contraceptives; Fertility

\section{Chhabra S*}

Department of Obstetrics Gynecology, Mahatma Gandhi Institute of Medical Sciences, Sevagram, Wardha, Maharashtra, India

\section{*Corresponding author: Chhabra S \\ - chhabra_s@rediffmail.com}

Department of Obstetrics Gynecology, Mahatma Gandhi Institute of Medical Sciences, Sevagram, Wardha, Maharashtra, India.

Tel: 917152284342

Citation: Chhabra S (2018) Possibilities of Planned Small Families in Communities with Lack of Awareness and Scarce Resources. J Contracept Stud Vol.3 No.1:5

Received: November 30, 2017; Accepted: January 04, 2018; Published: January 11, 2018

\section{Introduction}

It has been reported that each day, more than 800 women die due to pregnancy and birth related causes, which are preventable [1]. Many of these deaths could be prevented, if women had access to birth control, if they could prevent unwanted pregnancy and could increase the interval between two pregnancies. Based on a modeling exercise, Ahmed et al. [2] reported that, contraceptive use averted approximately 44 percent of maternal deaths. An additional 104,000 deaths could be averted annually by providing 
modern family planning methods to the 225 million women, who have an unmet need. Data from 27 low and middle income countries revealed that two thirds of women who desired postponing pregnancy for at least 24 months following birth, did not use modern contraceptive methods. Such women are at risk of becoming pregnant too quickly after a previous pregnancy, leading to increased risk of complications and death. The sustainable development goals (SDGs) (http://www.undep.org/ content/undp/en/home/sdgoverview) need to tackle the issue of unmet needs of family planning and aim at "universal access to sexual and reproductive health, by integration of reproductive health, including family planning information and education, into national strategies and programmes" [3]. Contraception is one of the proximate determinants of fertility and the most important predictor of fertility transition [4]. The choice of the contraceptive method, however, is influenced by a host of interdependent demographic, cultural, economic, and social factors which means that a multidimensional approach needs to be adopted for analyzing the contraceptive use. Modalities need to be tailored to the needs of the communities, understanding the ground realities. It may be that due to extreme poverty, the couples just do not think about planned small families, advantages of small family for better life for themselves and their children. Probably they just struggle for everyday survival, food, shelter and clothes. So they have large families and the vicious cycle goes on, more pregnancies, more abortions, more births, more babies and more deaths of mothers and babies (www. mayoclinic.org>ort20045756). They need creative awareness. Normally health professionals do talk about family planning knowledge, attitude, and practices, but linkage between everyday life, reproductive life, and family planning practices is not clear.

\section{Objectives}

To get information related to possibilities of small families in communities with extreme poverty.

\section{Methodology}

Information was collected by simple review of relevant literature, studies, reviews, commentaries and personal observations were added.

\section{Results and Discussion}

While integration of family planning services into maternal health programs and availability of contraceptive methods are keys to promoting women's health, the barriers to contraceptive use must be examined. Even when family planning services are available, many women do not utilize them for various reasons. Reported barriers to efficacy of integration of family planning into postpartum services include, weak counselling skills among postpartum staff, lack of job aids to increase patient awareness, lack of privacy for counselling and lack of husband's and motherin-law's involvement in the counselling process. Preconceived notions about health services also impact a woman's decision to seek care. Even with sufficient supplies and staff to counsel women, these barriers significantly impeded the impact of welldeveloped and well-integrated programs. Overcoming these barriers means providing accessible care, culturally-sensitive and patient-centered [5]. In a study it was revealed that women were more likely to adopt a contraceptive method of their choice and less likely to become pregnant at the 12- and 18-month period post-discharge with quality improvement strategies weaved into Family Planning (FP) maternal health integration efforts.

It is not very well known whether people with extreme poverty really want to have large families, especially when it is obvious, that limited resources make their everyday survival difficult. It is not very well known whether they would like to take action for safe future for themselves, their children and if so, what will they like to do and how? Mothers living in extreme poverty start working at home in a day after birth, move to work in fields within 2 to 3 days of birth, for their own and their family's survival. Each day's earnings are important for family's survival. It leads to loss of lactation as a method of spacing. Breastfeeding gets affected. When asked why they do this, the answer is 'no money, no food'. They do not have the understanding of breast milk storage. Also there are no resources for breast milk substitutes. So baby suffers. Further with breastfeeding, baby gets protection against infections also. Breastfeeding is believed to increase intelligence, probably reduces chances of overweight and diabetes. If there is no breastfeeding, woman's own health gets affected, because of frequent pregnancies and births. In addition to advantages of improving birth spacing, breastfeeding gives protection against breast cancer, may be against ovarian cancer and type 2 diabetes also [6]. However, how much is the awareness of breastfeeding as a method of birth spacing and other advantages of breastfeeding to woman's and baby's health in communities with extreme poverty, where child births are also more, is not well known. In many communities in India, there are rituals after birth, with isolation of mother and child, with ceremonies at 6-7 days and/ or 3 weeks or 40 days (equal to 6 weeks puerperium) after birth, depending on local culture. It seems that the most common family planning method is sterilization, after being pushed into it by health professionals or others, with targets.

So it is not surprising that while India was the first country to have an official family planning program in 1952, Indian population continues to grow, with lack of planned small families in many parts of the country. During its early years, the programme focused on the health rationale of family planning. Family planning as a strategy for population stabilization in India received attention only after 1971 population census [7]. However, the family planning programme has remained dynamic, after the historic initiation, with many transformations in terms of policies and actual programme implementation with a gradual shift to the reproductive child health approach. The National Population Policy (NPP) in 2000 brought a holistic and a target free approach which seems to be helping in the reduction of fertility controversy surrounding the popularity of permanent methods, for which women may be pressurized due to financial crunch in family or the open/hidden targets of service providers, continues. After the launch of the National Rural Health Mission in 2005, the official family planning programme has been subsumed in the reproductive and child health component of the Mission [8]. However, universal adoption of small family norm still remains 
a distant dream. During 2007-2008, only about 54 percent of the married women aged 15-49 years or their husbands were using a contraceptive method to regulate fertility and the contraceptive prevalence rate (CPR) appeared to have stagnated after $2004[9,10]$. Contraceptive practices are known to be very heavily skewed towards terminal methods, which mean that contraception in India is practiced for birth limitation rather than birth planning. According to District Level Household and Facility Survey (DLHS)-III, all the spacing methods together account for just around $25.5 \%$ of the current contraceptive use compared to $74.5 \%$ by female and male sterilizations put together (Figure 1).

May be even when family planning services are available and women know, they do not use contraceptives for various reasons. In communities with extreme poverty with lack of access, lack of awareness persists; it could be influence of their own past experience of side-effects or the experience of others in their social circles, and cultural beliefs. One woman's negative experience may be enough to dissuade her, her family, neighbors or friends and the whole community. In many settings, fear of undesirable effects has proved to be an important deterrent to seeking contraceptives. Many women tend to pick methods if approved by husband and sometimes by mother-in-law also, which conventionally holds a high place in the Indian family structure. Many also believe that oral forms of birth control can cause infertility. Preconceived notions about health services also impact a woman's decision to seek care [5]. It has been reported that women who receive comprehensive and factual education regarding family planning are more likely to use contraception. Women who cite methods with greater side effects or negative cultural perceptions are less likely to use them. DLHS-III [11] revealed that the knowledge of family planning methods is almost universal in India, with 99 percent women reporting the knowledge of one method or the other. Among traditional methods, rhythm method was the most popular method of family planning. CPR for some modern methods was quite high in some states. Pill was the most popularly known method among the temporary modern methods of family planning. At the national level, 53.3\% married women were aware of rhythm method. Among the permanent modern methods, female sterilization was the most popular, (35.8 percent women opted for sterilization). The government health facilities, government/municipal hospitals, community health centres, primary health centres and sub-centres were the major sources of contraceptives. Further, the DLHS-III also revealed that $93 \%$ of sterilization was paid for by the government, while only $47 \%$ of oral contraception was paid for by the government. This unequal distribution of funds could also be a possible cause of the trend of women undergoing sterilization, instead of using a less permanent method. If women were using a reversible form of birth control, $69.4 \%$ discontinued due to fertility reasons, $11.5 \%$ due to side-effects and $19.2 \%$ due to others like low supply, cost, husband said no, and inconvenience [12]. Further it was revealed that the percentage of women who sought sterilization rose markedly after they had 2 children, from $5.1 \%$ to $43.3 \%$. However, the percentage of women seeking sterilization with no sons remained negligible, regardless of the number of children. Birth spacing in India also showed preference for sons. The median birth interval was shorter if the previous child was a girl, than if it was a boy, but the difference was only 0.4 months $[13,14]$ (NFHS-3). Unmet need of family planning was 21.3\% as per DLHS-III of the 6.4 million pregnancies that occurred in the United States, in 2001, 49\% were unintended. Of these 3.1 million unintended pregnancies, 52\% (1.6 million) occurred in women who were not using contraception during the month of conception, whereas the remaining $48 \%$ were contraceptive failures [15]. Although 'unintended' does not always mean unwanted pregnancy, these pregnancies should nonetheless be considered family-planning defeats. Apart from the emotional burden, unintended pregnancies are expensive for society in terms of medical cost, the cost of caring for more children [16].

Very little is known about the reasons behind differing contraceptive preferences and choices of different groups of women. Nearly $30 \%$ of women who stop using contraception for reasons other than wanting to get pregnant, cite concerns related to health service quality including lack of method choice, stock-outs, and ineffective referral systems. Delaying the age of first pregnancy would reduce maternal and newborn mortality as well as improve the health and well-being of young girls [17].

Analysis of the 2008 National Demographic and Health Survey (NDHS) data showed that children ever born (CEB) were inversely related to education, modern sector work and family wealth. Women from the poorest families tended to have the most number of children, as they gave birth at a young age and continued childbearing beyond age 40. Moreover, they were also less likely to use a contraceptive method and more likely to have unmet family planning need. The high fertility among the poorest segment of the population has significant implications on social welfare of the individual families and socio-economic development at the macro level. Reasons given by the respondents for non-use of contraception suggest that accessibility of family planning services is not a barrier to contraceptive use even among the poorest and it is possible that other factors resulted in the relatively low CPR.

However, in regions with extreme poverty, many things are not known, including women's awareness about spacing methods.

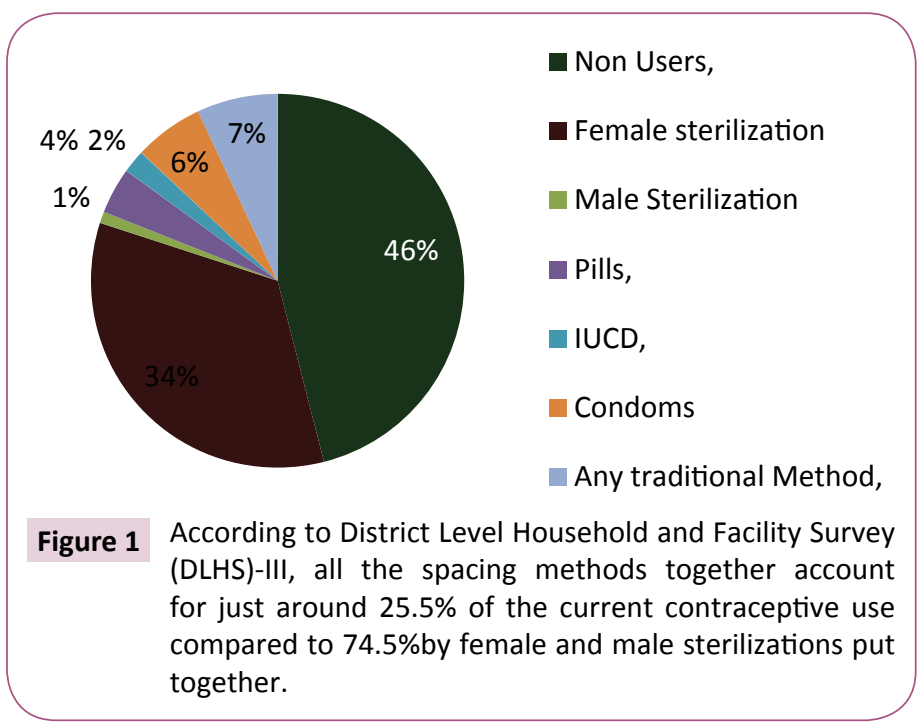


In such situations exposure to health settings and health professionals, which influence family planning practices, is also limited, due to scarcity of accessible equipped care, lack of basic prenatal care, and high rates of home deliveries [18].

A recent study carried out in five states of India has, however, revealed that there is substantial demand for contraception among young married women to postpone their first pregnancy, but only a small proportion of these women use any contraception [19]. It is, however, not clear, how much of the unmet need of contraception among young married women is due to the lack of resolve to practice contraception and how much of the unmet need is due to supply side factors. The pattern of contraceptive use has been found to be highly sensitive to household standard of living, irrespective of the number of surviving children and religion. The rich-poor gap in contraceptive use is large because the use of modern spacing methods is more than four times higher in women belonging to households, with very high standard of living compared to women belonging to poor standard. It reflects the fact that access of women to modern spacing methods of contraception increases with the increase in the household standard of living. At the same time, it may also be argued that women belonging to households with low to very low standard of living may be interested in producing the desired number of children quickly and then go for a terminal method to stop childbearing, because they may not have the capacity to plan or space the family either because of the lack of knowledge or because of family and society pressure or because of the inability of the family planning service delivery system to ensure regular, uninterrupted supply of modern spacing methods. Obviously, any effort to encourage women belonging to households with low resources, to space births must be associated with an efficient family planning service delivery system. If the efficiency of the system is poor, any effort to embrace women to space births may largely remain nonproductive. Pachauri [20] opined that despite a long history, spanning some five decades, India's family planning programme has not yet achieved replacement level fertility. Chaurasia [21] reported poor efficiency of the family planning services delivery system in the country to ensure supply of modern spacing methods of contraception especially to women belonging to households with low to very low resources and limited influence of schooling of women and their husbands on contraceptive use. Chipeta et al. [22] reported that despite knowing about the different types of family planning methods, and awareness of their ready availability in health facilities, use of these methods was low because considerable misinformation prevailed regarding contraceptive methods' side effects, factors that affected the intentions of men and women to use family planning methods. A program's ability to be effective depends upon its capacity to address the needs of the couples, it is designed to serve and promote an environment of respectful and user-centered care [5].

There is evidence that the most effective strategies to prevent unintended pregnancies are education, appropriate counseling regarding all available contraceptive methods, and provision of the most effective methods [23].

\section{Conclusion}

It is essential to provide comprehensive guidance to service providers [24]. It is essential to explore, information about the factors that influence women's/couples' willingness to use family planning with concept of sustainability in communities with extreme poverty for planned families, limiting family size with proper spacing. It is essential to know whether couples/women with extreme poverty ever think about planned small family. If no, why, if they think, what do they do? Do they know about family planning modalities, what do they know? Which family planning methods are accessible to them? Why a particular method is used or not used? What are the bottlenecks in use? What are their post birth practices, about work away from home, feeding of the newborn and contraceptive use? When is breastfeeding, begun? What is the duration? Do they know about breast milk storage? Do they know that exclusive breastfeeding prevents pregnancy? Do they know about other advantages of breastfeeding to the baby and to the mother? What are their post birth contraceptive practices? Do they do anything? What is the interval between birth and contraception, type of contraception, duration and so on? It is essential to go in depth about their everyday life, reproductive life, before one goes into advocacy of various issues related to small planned family, possible modalities of prevention of pregnancy and birth. It is essential to know issues which directly affect, planning of family, contraceptive use, traditional methods of their choice like withdrawal, rhythm, others vs. modern, (condom, female condom, intrauterine device, oral contraceptive pill, emergency contraception, injectable), reversible vs. long term. Also it is essential to know about issues which indirectly affect, number, status of living children, number of sons, termination of pregnancy if any, still births, desire to conceive; age at first pregnancy, number of pregnancies, family structure, size, role within family, maternal health and financial resources. Providing culturally sensitive and family centered, accessible modalities is the only sustainable way. There is need of successful promotion of women centered environment that respects their beliefs. A program's ability to be effective in the community depends upon its capacity to address the needs of the women/couples, it is designed to serve and promote an environment of respectful and community centered care with their perceptive weaved into [5]. There is need of flexible models that are most responsive to the needs of women/couples [17]. Advocated that family planning counseling/advocacy must be an essential part of antenatal and postnatal care and a dialogue about contraception should be part of any clinical encounter with women of reproductive age. From the policy and programme perspective, in the context of universal access to family planning services, there is a need to enhance the needs effectiveness and increase the capacity, efficiency of family planning efforts, so that they address specific family planning needs of couples with distinct social, economic, and personal characteristics. It is the need of the time that family planning efforts in India explore the determinants of different contraceptive preferences and choices across different groups and reinvigorates themselves to address these preferences and choices in an efficient, yet cost effective 
manner [21]. In order to deliver family planning information and services to couples who want and need it, programs must tailor their services to match the cultural values, unmet needs, and literacy levels of their target audience. These factors vary between and within countries, which means that each program needs to be sensitive to the unique characteristics of its target population. Successful integration of family planning services

\section{References}

1 Tobey SA, Swaim L, Clark S (2015) The role of obstetrics/ gynecology hospitalists in reducing maternal mortality. Obstet Gynecol Clin N Am 42(3): 463-475.

2 Ahmed S, Li Q, Liu L, Tsui AO (2012) Maternal deaths averted by contraceptive use: an analysis of 172 countries. The Lancet 380(9837): 111-125.

3 Millar K (2016) MHTF Quaterly, 7: Family Planning and Maternal Health.

4 Lai S, Tey N (2014) Socio-Economic and Proximate Determinants of Fertility in the Philippines. World Applied Sciences Journal 31: 1828-1836.

5 Flores ES (2016) MHTF Blog. Beyond availability: Barriers to contraceptive method use.

6 Victora C, Bahl R, Barros A, França GVA, Horton A, et al. (2016) Breastfeeding in the 21st century: epidemiology, mechanisms, and lifelong effect. The Lancet 387(10017): 475-490.

7 Chaurasia AR, Gulati SC (2008) India: The State of Population 2007, Government of India, national population commission and Oxford University press, New Delhi, India.

8 Chaurasia AR Singh R (2013) "Forty years of planned family planning efforts in India," in Proceedings of the 2013 IUSSP International Population Conference, Bussan, Republic of Korea.

9 United Nations, Update for the MDG Database: Contraceptive Prevalence, Department of Economic and Social Affairs, Population Division, New York, NY, USA, 2012.

10 Family Planning 2015-16.

11 International Institute for Population Sciences, District Level Household and Facility Survey (DLHS-III), 2007-08, IIPS, Mumbai, India, 2010.

12 Diamond-Smith N, Campbell M, Madan S (2012) Misinformation into maternal/reproductive health program and simultaneously promotion of an environment that respects the target population's values is essential. Efforts are, therefore, currently underway to reconceptualise the programme to design and implement priority strategies for achieving programmatic goals integrated programmes, that can more effectively and efficiently address multiple reproductive health needs of the people.

and fear of side-effects of family planning. Cult Health Sex 14(4): 421-433.

13 National Family Health Survey (NFHS-3); fertility and fertility preferences.

14 Family Planning. Annual Report 2015-16.

15 Finer LB, Henshaw SK (2006) Disparities in rates of unintended pregnancy in the United States, 1994 and 2001. Perspect Sex Reprod Health 38(2): 90-96.

16 Bartz D, Greenberg J (2008) Sterilization in the United States. Rev Obstet Gynecol 1(1): 23-32.

17 Caglia J (2016) MHTF Blog, Family planning and maternal health: Four aways from the International Conference on family planning.

18 Babu G, Ramachandra S, Garikipati U, Mahapatra T, Mahapatra S, et al. (2012) Maternal Health Correlates of Neonatal Deaths in a Tribal Area in India. Int J Epidemiology 10.

19 Jejeebhoy S J, Santhya KG, Zavier AJ (2014) "Demand for contraception to delay first pregnancy among young married women in India." Stud Fam Plann 45(2): 183-201.

20 Pachauri S (2014) Priority strategies for India's family planning programme. Indian J Med Res 140(1): 137-146.

21 Chaurasia A (2014) Contraceptive Use in India: A Data Mining Approach. Int J Population Res 2014: 1-11

22 Chipeta EK, Chimwaza W, Kalilani-Phiri L (2010) Contraceptive knowledge, beliefs and attitudes in rural Malawi: misinformation, misbeliefs and misperceptions. Malawi Med J 22(2): 38-41.

23 Secura GM, Madden T, McNicholas C (2014) Provision of nocost, long-acting contraception and teenage pregnancy. $\mathrm{N}$ Engl J Med. 371: 1316-1323.

24 Black (2015) Canadian contraception consensus (Part 2 of 4). J Obstet Gynaecol Can 37(11): 1033-9. 